\title{
La seguridad y salud ocupacional a través de los años
}

\author{
Occupational health and safety through the years
}

\author{
Atziri W. Sánchez-Oropeza ${ }^{a}$, Isidro J. González-Hernández ${ }^{b}$, Rafael Granillo-Macías ${ }^{c}$, \\ Zaraeli Beltrán-Rodríguez ${ }^{d}$, Leilani Ramírez-López ${ }^{e}$, Brayan Sotero-Montalvo ${ }^{f}$
}

\begin{abstract}
:
Occupational Safety and Health is a multidisciplinary field that includes the recognition, prevention, and control of risks and dangers in the workplace to promote workers' safety and health. This discipline is the result of years of research and professional maturation of four drivers: best labor practices; certifications and education standards; laws, policies, and accidents; and technological advances. Therefore, this work aims to present the historical evolution of occupational health and safety through a qualitative description of a bibliographic and documentary nature to identify the challenges that existed in this discipline in industrial revolutions and identify which are the new ones. Industry 4.0 technologies that are being used for Occupational Health and Safety Management Systems. The studies analyzed reveal that the techniques, procedures, and legislation on occupational safety and health throughout the world were limited compared to the technological developments that were generated in the first two industrial revolutions. In the context of the fourth industrial revolution, it was identified that the two most important risk factors that will constantly be present in the work areas are mental fatigue and psychological pressure. However, Industry 4.0 technologies such as the Internet of Things, Big Data, and advanced or intelligent sensors are being used to build platforms that allow predicting workplace accidents.
\end{abstract}

\section{Keywords:}

Occupational health and safety, risks, technology

\section{Resumen:}

La Seguridad y Salud Ocupacional es un campo multidisciplinar que comprende el reconocimiento, la prevención y el control de riesgos y peligros en el lugar de trabajo con el fin de promover la seguridad y salud de los trabajadores. Esta disciplina es el resultado de años de investigación y maduración profesional de cuatro impulsores: mejores prácticas laborales; certificaciones y estándares de educación; leyes, políticas y accidentes; y avances tecnológicos. Por lo tanto, el objetivo de este trabajo es presentar la evolución histórica de la seguridad y salud ocupacional mediante una descripción cualitativa de carácter bibliográfico y documental para identificar los desafíos que existían de esta disciplina en las revoluciones industriales, así como identificar cuáles son las nuevas tecnologías de la Industria 4.0 que se están empleando para los Sistemas de Gestión de Seguridad y Salud en el Trabajo. Los resultados revelan que las técnicas, procedimientos y la legislación sobre seguridad y salud ocupacional en todo el mundo estuvo limitada en comparación de los desarrollos tecnológicos que se generaron en las primeras dos revoluciones industriales. En el contexto de la cuarta revolución industrial, se identificaron que los dos factores de riesgo más importantes que se presentarán constantemente en las áreas de trabajo son la fatiga mental y la presión psicológica. Pero las tecnologías de la Industria 4.0 como el Internet de las Cosas, Big Data y los sensores avanzados o inteligentes se están utilizando para construir plataformas que permitan predecir accidentes laborales.

\section{Palabras Clave:}

Seguridad y salud ocupacional, riesgos, tecnología

\footnotetext{
a Universidad Autónoma del Estado de Hidalgo, https://orcid.org/0000-0002-1896-9528, Email: sa408599@uaeh.edu.mx

b Universidad Autónoma del Estado de Hidalgo, https://orcid.org/0000-0003-2805-6674, Email: igonzalez@uaeh.edu.mx

c Universidad Autónoma del Estado de Hidalgo, https://orcid.org/0000-0002-1015-667X, Email: rafaelgm@uaeh.edu.mx

d Universidad Autónoma del Estado de Hidalgo, https://orcid.org/0000-0001-9221-4302, Email: be334291@ uaeh.edu.mx

e Universidad Autónoma del Estado de Hidalgo, https://orcid.org/0000-0002-9593-5823, Email: ra404688@uaeh.edu.mx

f Universidad Autónoma del Estado de Hidalgo, https://orcid.org/0000-0003-2456-564X, Email: so405664@uaeh.edu.mx
} 


\section{Introducción}

En el presente entorno se encuentran todo tipo de situaciones de las que, su mera existencia se ha vuelto parte de un escenario y por ello, no hay reparo en saber qué son y de dónde provienen. Por decir, se entiende que todos los productos que están al alcance humano son producidos en enormes fábricas en donde intervienen diferentes máquinas que son operadas por personal capacitado, pero hasta ese punto llega el análisis del público consumidor; no hay consciencia plena de los factores que están involucrados en los procesos industriales y, sobre todo, de manera casi instintiva, lo último en lo que se piensa, además de saber el cómo se obtiene un producto, es el término de seguridad e higiene (seguridad y salud ocupacional).

Es un hecho que, si los mismos operarios, quienes son responsables de seguir las bases que dictan la seguridad e higiene, no le dan la importancia y cumplimiento necesarios, mucho menos lo hará el resto de la población que, en la mayor parte del tiempo, desconoce los procesos (Antezana, 2012).

De esta manera, la importancia de aplicar y cumplir con lo que la seguridad e higiene establece, radica en tener dominio del verdadero significado y con ello, comprender el impacto que ejerce en los entornos de trabajo, y más específicamente en el ámbito industrial.

Sin embargo, para analizar un elemento es indispensable conocer su origen, analizar cómo fue evolucionando hasta moldear el esquema presente y así, destacar el papel que juega la seguridad e higiene en el entorno industrial y/o empresarial.

Ahora bien, tomando en cuenta tales aspectos, es preciso comenzar a definir la primera interrogante: ¿Cómo surge la seguridad e higiene? ante esto, se desarrollan otras cuestiones relacionadas: ¿De dónde proviene?, ¿Cuándo comenzó a aplicarse?, ¿Por qué fue implementada? Y, finalmente ¿Cuál es la evolución de la seguridad y salud ocupacional a través de los años?

Aunque parezca sencillo responder a estos cuestionamientos, en realidad, al detenerse a analizarlos a profundidad, surge una enorme laguna en la historia de la humanidad, ya que es cierto que muchas de las cosas de las que se cuestiona su origen, están perdidas entre cientos o miles de años de datos e información, por lo que resulta casi imposible definir momentos y lugares exactos en los que acontecieron las bases sobre la seguridad e higiene.

No obstante, al profundizar en el tema y ahondar en los datos más sobresalientes, es posible encontrar resquicios históricos que pueden dar indicios de lo que se está buscando, por ello, pese a no tener con exactitud el lugar y momento en donde nace la seguridad e higiene, se tiene referencia de algunos de los primeros eventos que fueron establecidos y documentados para comenzar con los estudios que más adelante, darían paso al asentamiento del concepto y de cómo ha logrado ejercer un papel de suma relevancia en las actividades laborales que forman parte de la vida diaria en todos los sectores. Así pues, se comienza con el hecho de que la seguridad industrial se encarga de garantizar la ocurrencia mínima de riesgos laborales a fin de prevenir los accidentes que suceden durante el desempeño laboral; para ello, se entiende que los riegos laborales representan las probabilidades de sufrir lesiones (daños físicos), daños al medio ambiente, o bien, pérdidas en los procesos y equipos hablando de un entorno laboral (Arias, 2012).

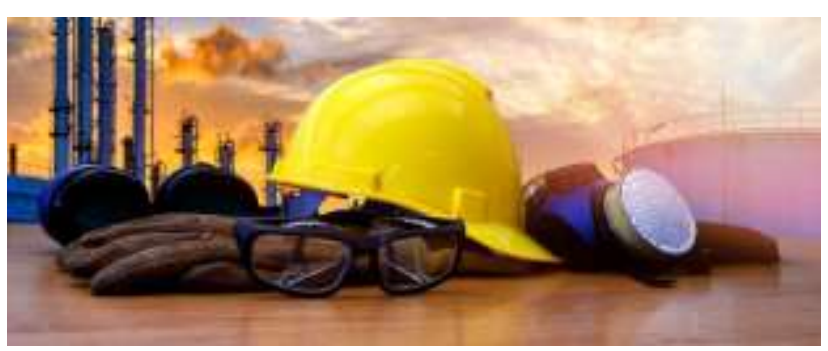

Figura 1. Seguridad en el trabajo (tomada de Ruiz y Choroco, 2019)

\section{Método}

La metodología de este trabajo incluye una investigación documental basada inicialmente en una revisión de la literatura sobre dos aspectos: 1) la evolución de la seguridad y salud ocupacional para identificar cuáles fueron las técnicas, procedimientos y la legislación sobre seguridad y salud ocupacional que se desarrolló a partir de la primera revolución industrial. 2) Identificar las tecnologías de la Industrial 4.0 que se están empleando en los sistemas de administración de seguridad y salud ocupacional.

\section{Evolución de la seguridad y salud ocupacional.}

Antes de entrar en materia de lo que implica la seguridad industrial y el papel que ejerce la salud ocupacional, es necesario situarse en los primeros vestigios de la humanidad, cuando el hombre descubría la utilidad de las cosas y ponía a prueba sus inexploradas habilidades para dar paso a nuevas y mejores formas de llevar a cabo las actividades de su día a día.

En tal caso, es bien sabido que el hombre, desde sus principios en la tierra como un ser racional, ha manipulado los recursos a su alcance y ha aprovechado lo que su entorno le brinda para hacer que su rutina fuera más llevadera a modo de no incurrir en esfuerzos físicos que derivan en labores arduas y realmente pesadas para 
el soporte del cuerpo humano. En este sentido, es correcto decir que el hombre aplicaba simples bases de ingenio que le facilitarán las acciones, ya que, como tal, no aplicaba un análisis tan riguroso en el que considerara todos los factores involucrados como la seguridad establece, sino que, el individuo, tras realizar una determinada actividad en cierto número de ocasiones, comenzaba a registrar un patrón de esfuerzo y evaluaba cuáles eran las mejores alternativas para poder hacer las cosas de manera más rápida, menos cansada y más eficiente. Estos indicios señalan que el hombre ya tenía en consideración la importancia de restarle esfuerzo a sus actividades, aunque, no por ello resulte correcto decir que sucedió lo mismo con la medición de riesgos, ya que el hombre, aun considerando el esforzarse menos, no tenía un control preciso sobre salvaguardar su salud o su vida misma, esto, debido a que no se tenía plena consciencia de que algunas actividades implicaban un verdadero peligro y por ello, continuaban realizando lo mismo, una y otra vez, sin pensar ni accionar una estrategia que erradicara los focos de riesgo (Bernal, 2013).

De tal forma, con el pasar de los años la humanidad comenzó a crecer a lo largo y ancho del mundo hasta situarse en un periodo indistinto en el que las poblaciones pasaron a conformar una sociedad compleja en la que se dio la adquisición de poder y riquezas de unos sobre otros; así mismo, surgen las estratificaciones de ideologías totalmente subjetivas en las que, se estableció que el valor de una persona estaba dado por la cantidad de riquezas que poseía, o bien, por la cantidad de conocimientos que albergaba. Ciertamente, aquellos que no poseían estaban destinados a trabajar para otros con el fin de poder vivir, con lo cual, se designó el hecho de que las labores manuales eran la parte más inferior de los trabajos y que por ello, no representaban valor alguno. Siendo así, con los complejos de valor entre unos y otros, hay un buen tramo de historia resumido en la construcción de los grandes imperios y civilizaciones que representan todo un complejo de situaciones en los que se vivieron calamidades de todo tipo; aquellos que no poseían valor alguno entre las escalas sociales, eran tratados como la misma nada y por ello, su vida no representaba relevancia de ningún tipo; en concreto, los trabajadores debían tolerar todo tipo de abusos, maltratos, situaciones insalubres y peligros por parte de aquellos que los obligaban a realizar tareas que representaban esfuerzos físicos casi inhumanos, en su mayoría estaban resignados a tolerar esos abusos por que tenían complejos sobre sí mismos como seres que no poseían nada y que por ello, su destino estaba ligado a servir a otros; no obstante, aquellos que no estaban sumidos en continuar con esa pesadilla tomaron dos rumbos: alzar la voz para esforzarse por frenar la situación y ser silenciados en su intento o bien, profundizar en el tema y tomar datos de lo que acontecía a su alrededor, estudiar las causas, los efectos y las medidas que podrían servir de utilidad para disminuir los daños en su entorno y en su persona misma (Arias, 2012).

Pese a que el tiempo no sucumbía tan rápido como las personas, los cambios se hicieron notorios y pronto comenzó a florecer el sentido de realizar las actividades bajo los menores riesgos posibles; en su debido tiempo, lugares como Mesopotamia, Israel, Egipto, Grecia y Roma, así como Francia, Inglaterra, Alemania y Estados Unidos, destacaron por su implementación de medidas en lo referido a seguridad y salud ocupacional con la inserción de estrategias que ni bien, tenían el objetivo de facilitar las actividades laborales al tiempo de disminuir la ocurrencia de daños físicos que representaran dificultades o retrasos en su ritmo laboral.

Así pues, surgen los primeros equipos que, pese a no ser por objeto de salvaguardar la salud e integridad de los trabajadores, eran eficientes al disminuir el grado de dificultad o cansancio de las labores; por mencionar, la inclusión de sandalias fue con el objetivo de que los trabajadores pudieran permanecer más tiempo de pie en comparación a estar descalzos en superficies difíciles de soportar; así mismo, el uso de arneses y andamios permitían trabajar a grandes alturas sin correr el riesgo de desperdiciar material o tiempo (Arias, 2012).

A su vez, hay registros que indican el surgimiento de numerosos estudios encargados de analizar de manera más ardua y objetiva, las causas de las enfermedades, intoxicaciones por zonas determinadas de trabajo, decesos del personal, los accidentes, las posturas incómodas, las causas de fatigas, las deformaciones físicas, el control sanitario y con ello, las medidas que debían ser consideradas para que el trabajador pudiera desempeñar sus labores bajo el menor riesgo o peligro. De entre tantos, el que mayor destaca es Bernardino Ramazzini (Carpi, 4 de octubre de 1633 - Padua, 5 de noviembre de 1714) a quien se le considera el padre de la salud ocupacional, aun cuando ésta no había sido formalmente definida (Hofmann, 2017). 


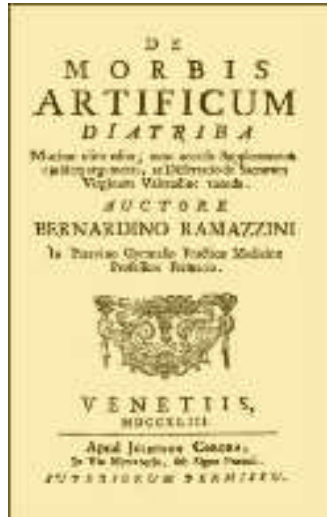

Figura 2. De morbis artificum diatribe Ramazzini (tomada de Fresquet, 2011)

De este modo, se da entrada a un paso histórico notable; las revoluciones industriales, que bien, es cuando la seguridad industrial ingresó de manera formal al marco histórico de su estudio; es bien sabido que con la llegada de las nuevas tecnologías se generó un cambio importante en el sector laboral y con ello, en el mismo entorno social. Ante el crecimiento industrial y el desarrollo de la economía, surge la necesidad de conseguir oportunidades de crecimiento, vidas más prometedoras y con ello, la esperanza de un futuro mejor alejado de la miseria y la vida simple del campo; en este sentido la gente del campo abandona sus hogares con ambientes y condiciones seguras para huir a la ciudad que ya estaba engullida por el caos industrial, la seguridad no tenía un papel en la jugada y con ello, se propician la explotación y la esclavitud laboral; las enfermedades aumentan junto con el número de muertes por las condiciones laborales en estado deplorable e insalubre, llevadas a cabo sin ningún tipo de protección ni bajo las medidas mínimas necesarias de higiene (Arias, 2012).

Ahora bien, destacando los sucesos del siglo XVIII, resaltan los estudios y documentaciones en base a las alarmantes situaciones de muerte y enfermedades por actividad laboral, que de forma considerable, han llamado bastante la atención y por ello, comienzan a dictarse reglamentos con el fin de proteger a los trabajadores a modo de otorgarles seguridad en su labor bajo los actos de disminuir el tiempo de las jornadas laborales, la fijación de niveles mínimos de higiene y salud, a la vez de adoptar paso a paso, medidas de seguridad más concretas, estableciendo inspecciones con el fin de verificar que las medidas están siendo respetadas y que con ello, se obtuvo mayor centro de vigilancia en el proceder de la seguridad de los trabajadores a modo de generar un ambiente laboral más estricto, aplicando estándares que se encargaban de preservar la ventilación para los lugares cerrados y con fácil obstrucción de aire, señalamientos para guiar a los trabajadores en las distintas áreas, válvulas de escape y frenos que permitieran manipular la máquina en todo momento, a fin de prevenir los accidentes en la mayor medida de lo posible. Sin embargo, es importante recordar que, pese a que la labor es reconocida y con ello, la vida misma del trabajador, no se puede olvidar que los trabajadores continuaban siendo vistos como un elemento más de trabajo que tenía tanta importancia como las máquinas y herramientas, pero no por ello, más importante que la producción misma (Arias, 2012).

Por otro lado, la búsqueda por implementar medidas y condiciones más adecuadas para las actividades, dado que el trabajo se vuelve una labor permanente en las actividades diarias de la sociedad y que, por ello, requiere de una normativa eficiente sobre derechos, deberes, prohibiciones y beneficios a los que deben sujetarse tanto los empleados como los empleadores; dio pie a que surgieran eventos de gran importancia como lo fue el responsabilizar a las empresas por los accidentes laborales (Hudson, 2019).

Así pues, se otorga mayor grado de responsabilidad y toma de medidas cada vez más estrictas por parte de patrones y contratistas que, de no actuar en favor de preservar la seguridad de sus trabajadores, tendrían severos problemas ante los mandatos establecidos (Antezana, 2012).

De tal modo, los diversos preceptos y tratados fueron evolucionando con el tiempo, a fin de dar estabilidad a los trabajadores que iban en busca de mejores condiciones y oportunidades, con ello, se destacan algunos sucesos tales como: el surgimiento de la Ley de 1833 que creó la Inspección de Fábrica en el Reino Unido con el objetivo de controlar el bienestar de los empleados y regular las condiciones de trabajo. En Estados Unidos, en 1910 se aprobó la ley de compensación a trabajadores que resultaran lesionados, haciéndose cumplir el concepto de responsabilidad de los empleadores (Salvador, 2016).

Con la creación de la Organización de las Naciones Unidas en el año de 1919, se da paso a internacionalizar las leyes en el ámbito laboral; tal como el Tratado de Versalles (postulado ese mismo año) con el que se da paso a la creación de la Organización Internacional del Trabajo (OIT) con el fin de velar por los derechos y la seguridad de los trabajadores a modo de otorgarles condiciones más humanas y justas para desempeñar su labor.

Por otro lado, de manera concisa se daba por hecho que las políticas periódicas tenían la intención de ofrecer adaptaciones a la fuerza laboral de oprimidos a fin de hacer arreglos hacia una perfección de las condiciones de trabajo dentro de la fábrica, dentro de todo esto, se obtiene que: en la India, la Dirección General del Servicio de Asesoramiento de Fábricas e Institutos Laborales estableció en 1947 la Ley de conflictos laborales, en 1948 
se crean la Ley de fábricas, así como la Ley de salario mínimo, en 1956 se da la Ley de sociedades y alrededor de 1986 surgen reglas para trabajadores portuarios (Singh, 2016).

Por otra parte, En Estados Unidos, en el año de 1990 surge una Ley de programación sobre la prevención de la contaminación en la que se establecen prohibiciones para la eliminación de desechos tóxicos, dando paso a regulaciones como la Ley de Conservación y Recuperación de Recursos (RCRA, por sus siglas en inglés) que funge al establecer límites permitidos de emisiones ambientales incluyendo medidas preventivas y correctivas con el personal que maneja el derrame de desechos (Perl, 2016).

Así como el caso dado a través del Convenio No. 161 de 1985 de la Organización Internacional del Trabajo (OIT) y el Plan de acción mundial 2008-2017 de la Organización Mundial de la Salud (OMS), destacados por ser entidades que demuestran el valor que la comunidad internacional brinda en cuestiones de seguridad con motivo de prestar servicios de seguridad y salud en el trabajo en virtud de las comunidades obreras (Ncube, 2018).

Eventualmente, surgen individuos meramente interesados en investigar tipos y causas de los accidentes en industrias destacadas, así como la asistencia sanitaria y la minería, mientras que, por otro lado, algunos tendían a explorar la seguridad y salud en el trabajo dentro de las condiciones de los límites nacionales, como las distribuciones de los riesgos entre los inmigrantes y los no inmigrantes en países con políticas migratorias favorables (Fan, 2020).

Dicho de otro modo, los obreros dejaron de verse como un elemento más del entorno laboral y comenzaron a contarse como lo que eran verdaderamente: personas con el mismo valor e importancia que los patrones mismos. Dado que la vida humana adquiere matices más concretos, términos como esclavitud y absolutismo comienzan a verse rechazados y con ello, la lucha por obtener mejores condiciones de trabajo es cada vez mayor; en todas partes del mundo se puede visualizar que la idea de manipular las máquinas y llevar a cabo los procesos de obtención y explotación de materiales, sin ningún tipo de protección es algo totalmente absurdo, se busca que los empleadores aseguren que la vida de los obreros no corra un riesgo potencial de muerte o de lesiones severas, que las condiciones de trabajo sean respetables con ambientes sanos, libres de infecciones, enfermedades o intoxicaciones, espacios en los que puedan desempeñarse hasta conseguir un nivel de producción deseado que no sea interrumpido por accidentes o catástrofes.

Llegando a este punto, se puede definir que el término de seguridad industrial adquiere mayor fuerza y consistencia en lo que respecta a los roles que cada individuo ejerce sobre la actividad productiva. Se ha generado tal nivel de apreciación sobre quienes poseen habilidades que sirven para mantener o aumentar las condiciones productivas y con ello, se destaca la importancia de brindarles un ambiente bajo las condiciones adecuadas; siendo el punto en el que juegan un papel relevante otro tipo de factores que están enlazados con la seguridad del trabajador; tales como la ergonomía, el manejo de desechos, la protección ambiental y los niveles de generación económica.

Ahora bien, al situarse en tiempos más cercanos, se encuentran asentamientos reglamentarios como lo es la norma ISO 31000:2018 que se rige bajo la gestión de riesgos en torno a tres etapas que incluye el análisis, la evaluación y el control de riesgos (Ramos, 2020).

De manera concreta, es posible entrever la adaptación que presento la seguridad y salud ocupacional, mezclándose de manera libre y racional entre los sucesos más destacables de la evolución humana, dejando huella en torno a su afirmación como un campo realmente importante que logra convertirse en el sustento de toda área involucrada en los sectores que componen los ámbitos laborales, específicamente, en el campo industrial. Se habla de un gran paso entre ser un resquicio de observaciones y medidas sugeridas; tratamientos, curaciones y trucos de posturas, a ser parte central de las actividades en las empresas en donde se consideran todos y cada uno de los aspectos que conforman el entorno laboral; iluminación, ventilación, manejo y control de maquinaria y herramientas, además de girar la atención en el individuo, lo que conforma al trabajador como una persona, un ser racional con fragilidad tanto física como emocional y no solo como un elemento más en la planta.

Se ha vuelto una realidad que la seguridad en la empresa, así como la salud ocupacional, son los pilares que han logrado posicionarse por encima de los intereses productivos, en conjunto a los aspectos que componen la economía, el medio ambiente y la sociedad; es más importante salvaguardar la vida de todos y cada uno de los individuos que se encuentran laborando en las empresas con el fin de seguir políticas sólidas en materia de salud y seguridad, a fin de mantener el mayor nivel posible de actividades productivas; pese a que, aún hoy día, hay aquellos que no comparten tal perspectiva y denigran el valor del factor humano como una herramienta más (Reis, 2020).

Ahora bien, la introducción de la salud y seguridad ocupacional en las empresas ha representado un impacto positivo en base a los estándares de un nivel organizacional que debe ser acatado por gobiernos, empleados y empleadores; logrando así, la implementación de Sistemas Integrados de Gestión que 
sean de utilidad en el manejo de control de riesgos que se mantienen latentes en todo momento (Ramos, 2020).

\section{Tendencias de seguridad y salud ocupacional en la Industria 4.0}

Bajo los estigmas asumidos en torno al funcionamiento de los procesos industriales, el paso de la historia y sus cambiantes paradigmas han dado pie a buscar en todo momento, la perfección en los métodos implicados para realizar cualquier tipo de labor; específicamente aquellos que implican facilitar las tareas más comunes. Es increíble hasta donde ha llegado el ingenio humano, de convertir máquinas de vapor a enormes complejos mecánicos que funcionan bajo sistemas computarizados. No obstante, las organizaciones en constantes ocasiones no han logrado asignarse de manera correcta para la implementación de nuevas tecnologías, con lo que se genera recelo en cuanto a la acción de la salud y la seguridad en el trabajo (Polak-Sopinska, 2020).

Es una realidad que la tecnología está avanzando más rápido de lo que lo hace la comprensión del hombre que, sin darse cuenta ha creado la base de la comodidad y facilidad de procesos bajo la simple acción de presionar un botón; cientos, miles de años de trabajos, abusos, muertes y esfuerzos por parte de los obreros que eran utilizados para las grandes producciones, se ha visto reducido a una monótona tarea de vigilar el buen funcionamiento de la máquina. Sumado a esto, la adopción de innovaciones como el internet con el fin de obtener soluciones eficientes, ha dado paso hacia la obtención de habilidades operativas con el propósito de proveer de un nivel estandarizado de información hacia el análisis de riesgos, generando un constante crecimiento en las actividades industriales, inmerso en las áreas responsables (Thibaud, 2018).

El control que los sistemas de inteligencia artificial representan en el buen funcionamiento y manejo de los procesos industriales, junto con los controles de prevención de riesgos en torno a patrones de análisis, se ha vuelto imperioso en la búsqueda de establecer las bases de todo proceso a fin de obtener resultados favorables: productos de calidad y buenas ganancias. Por ello, resulta altamente necesario que las empresas implementen nuevas o mejores tecnologías que les permita situarse en el linde de corporaciones exitosas que logran sus metas trazadas (Bortolini, 2020).

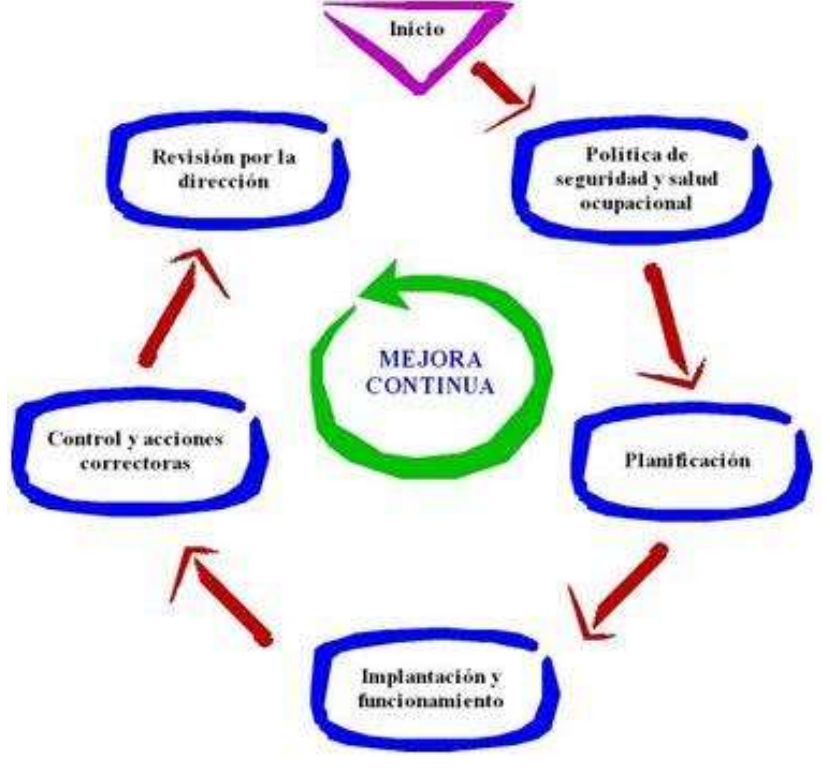

Figura 3. Sistema de gestión de seguridad y salud en el trabajo. OHSAS 18001:2007 (tomada de Espantelon,

2013)

De este modo, se logra abarcar un punto importante en relación a los medios y bases que las empresas deben implementar, por mencionar la inclusión de Sistemas de Administración de Salud, Seguridad y Medio Ambiente. Este tipo de sistemas de administración para la seguridad y salud ocupacional ha sido uno de los pasos más convenientes en cuanto a la recopilación de datos que permitan analizar e identificar los riesgos para generar soluciones en tiempo real por medio de patrones registrados. Sin embargo, la mayor parte de los sistemas convencionales de gestión de salud, seguridad y medio ambiente y de identificación de riesgos no tienen la capacidad de integrar datos de una forma ágil y automatizada o bien, de tomar decisiones inteligentes. Además, las bases de datos de estos sistemas suelen ser demasiado complejas de comprender, por lo que analizar la información depende de las destrezas personales de los individuos (Tarrahi, 2016).

Lo que lleva a un punto de retroceso sobre las implementaciones de sistemas que permitan cuantificar los riesgos durante las actividades laborales. Por ello, resulta preciso destacar que pese a mantener controles en la mayor medida de lo posible sobre la prevención de riesgos y fallas, la verdad es que resulta totalmente intangible suponer que realmente se puede tener un control absoluto en estas cuestiones, ya que hay una clara referencia de eventos que, pese a tener orígenes fáciles de rastrear y estudiar, el plano de los azares y las posibilidades es algo totalmente fuera de nuestro alcance; no existe total y pleno conocimiento sobre el momento y lugar exacto en el que va a ocurrir una falla o 
un accidente y por ello, es altamente necesario destacar en todo momento la importancia de seguir estrictamente todas y cada una de las medidas establecidas por la seguridad industrial (Adem, 2020).

Ya no se habla de establecer leyes o normas que les otorguen a los trabajadores las condiciones justas para realizar su labor, si no que ya es preciso establecer una idea concreta y transmitirla a todo el personal de los sectores sobre la vitalidad que ejerce el seguir las normas establecidas, respetar los parámetros y niveles permisibles de determinadas actividades bajo constante supervisión y, sobre todo; utilizar el equipo de protección personal en todo momento. Aplicando las herramientas necesarias que permitan lograr los objetivos establecidos por un plan de trabajo en el que se involucren proyectos y prototipos que brinden soluciones industriales a fin de desarrollar y aplicar el uso de las tecnologías (Gnoni, 2020).

Resulta importante definir que la modelización convencional de las causas de accidentes tiene limitaciones como el ignorar o simplificar factores clave, utilizar el análisis cualitativo y enfocarse en el análisis de la causalidad y las explicaciones de un accidente. Mientras que la tecnología de Big Data, gracias a su función de procesamiento paralelo y a su capacidad para manejar competentemente datos de gran dimensión y ruido con relaciones no lineales, resultará ventajosa y fructífera en el análisis de los riesgos para la salud y la seguridad (Ajayi, 2019).

Este tipo de tecnologías, con el desarrollo de modelos sólidos, logran ser efectivas para prevenir riesgos laborales como muertes, lesiones o incluso reducir el impacto ambiental en las empresas; además de darles mayor seguridad a los trabajadores en su respectiva zona de trabajo y producir una mejor toma de decisiones, basadas en el análisis de datos que permitirán prever incidentes a futuro.

Por otro lado, los wearables empresariales, otra de las tecnologías de la Industria 4.0, pueden fomentar una mejor higiene industrial para conservar a los operarios sanos, seguros y motivados dentro de los emergentes talleres inteligentes y sociales. ${ }^{8}$ Monitorear a un operador 4.0, definido como un operario inteligente y capacitado para realizar trabajo cooperativo y asistido por máquinas $y$ robots, y el entorno laboral que lo rodea en tiempo real es una tarea que se facilita gracias a los sensores portátiles y la inteligencia artificial. Convirtiéndose así, en la base de las aplicaciones y programas especializados para la salud y seguridad en el trabajo que buscan poder advertir a los trabajadores sobre riesgos ambientales, ergonómicos, paradas de emergencia y sobrecargas de trabajo tanto físicas como cognitivas.
El subtipo operador saludable 4.0 surge debido a la creciente preocupación por el aumento de estrés, riesgos físicos potenciales generados por los entornos con nuevas tecnologías y el estado de salud psicosocial de los trabajadores, puesto que utilizan soluciones inteligentes, como rastreadores, que incluyen capacidades de análisis de datos junto con tecnologías avanzadas de Interfaz Hombre-Maquina (HMI) y de Interfaz/Interacción Hombre Automatización (HAI), para utilizar sus datos biológicos (Romero, 2018).

Estas tecnologías, los wearables, pueden proporcionar un amplio catálogo de sensores que miden la aceleración, el movimiento y el estrés que se asocian a la carga de trabajo de los operarios; $y$ de esta manera buscar nuevas formas de medir la exposición del operador 4.0 a diversas fuerzas en tiempo real para buscar el bienestar ergonómico, de productividad, de salud y seguridad durante su rutina laboral.

Algunos ejemplos de estas tecnologías, emergentes de la Industria 4.0 en sistemas de manufactura, que ayudan al operador saludable 4.0 a tener salud, seguridad y una alta producción son:

- Los exoesqueletos inteligentes, son un tipo de dispositivo de asistencia portátil que ha sido integrado con sensores inteligentes en el cuerpo para el modelado biomecánico y de comportamiento con la intención de monitorizar y examinar en tiempo real los movimientos $y$ posturas corporales que no son ergonómicas, con el fin de evitar tensiones o lesiones en los operadores, mejorando la postura, reduciendo lesiones y esfuerzos físicos humanos que requiere el operador para realizar las tareas manuales. ${ }^{8}$ De esta manera se logra reducir el trabajo largo y agotador, además de la fatiga laboral. Estos dispositivos generarían una recopilación de datos continua de evaluación ergonómica de cada operario a lo largo del tiempo, apoyando a la creación de bases de datos de las tareas y posturas en las que se somete el operador para poder conocer y prever las condiciones de riesgos que se generan durante el trabajo.

- Los robots adaptativos, son robots colaborativos que se adaptan dinámicamente al ritmo, el nivel de estrés y la experiencia de los humanos. ${ }^{7}$ Representan un apoyo significativo para monitorear la labor cognitiva y física de los trabajadores; esto, debido a los sensores corporales inteligentes en el operador 4.0 que miden la conductancia de la piel, el movimiento del cuerpo y la frecuencia cardiaca, los cuales enviarán datos a un sistema de inteligencia artificial que se encarga de optimizar la ayuda 
cognitiva y física en función del operario. De esta manera el robot se adaptará a las necesidades cognitivas, físicas, rítmicas y demás a la salud del operador (Ajayi, 2019).

- Así mismo, la nueva generación de equipos de protección inteligentes, están impulsados por el desarrollo de wearables inteligentes y sensores de temperatura, humedad, ruido, luz, calidad del aire del entorno laboral en los lugares de trabajo industriales; además de los sensores de ubicación de movimiento y ubicación de los operadores. Estos nuevos equipos pretenden prevenir y garantizar de forma activa la salud y la seguridad de los trabajadores (Romero, 2018).

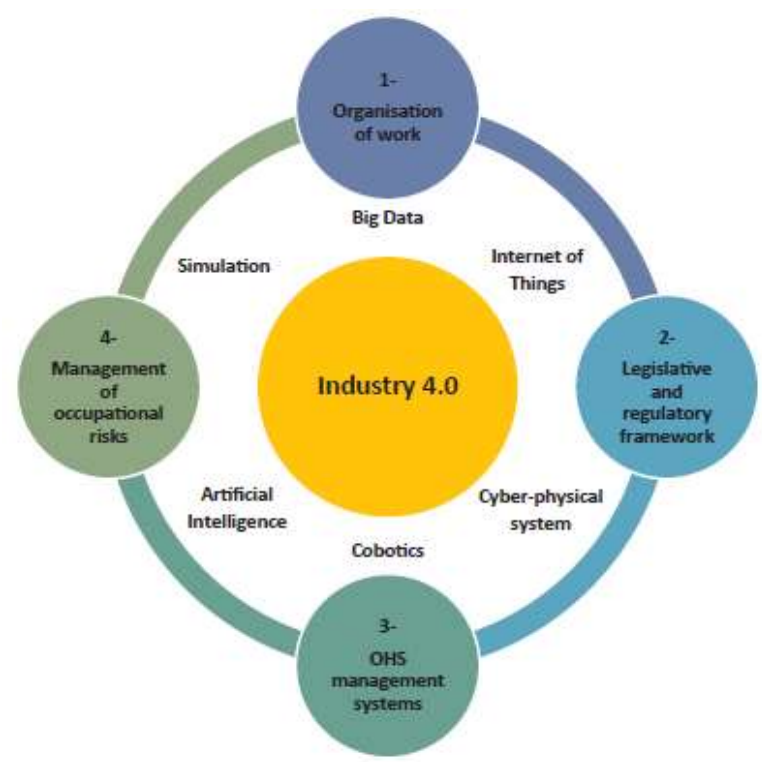

Figura 4. Tecnológicas de la Industria 4.0 y aspectos de la seguridad y salud ocupacional (tomada de Badri y Boudreau, 2018)

De entre estas y muchas otras tecnologías implementadas, que, a bien tienen por objeto el canalizar las condiciones de trabajo hacia una visión más propia y adecuada para los trabajadores; se vuelve al mismo punto de partida; el aspecto moral de los trabajadores. De manera concreta, poco o nada sirve el incluir y adaptar las más avanzadas tecnologías en términos de bases de datos y sistemas que permitan registrar la información referente al comportamiento sobre un análisis profundo de la ocurrencia de fallos y la trazabilidad de su corrección o prevención con el fin de promover la seguridad laboral; esto, debido a que el punto cúspide de lograr un entorno seguro en el ambiente laboral, va a ser definido por la capacidad de todo el personal involucrado (sean obreros, sean supervisores, sean empleados altamente capacitados) en seguir todos los lineamientos establecidos, tanto por las pautas definidas a nivel nacional e internacional, como por las medidas que la propia empresa establece con el fin de asegurar que todas las labores relacionadas con la actividad productiva no representen riesgos que atenten contra la salud física o emocional del personal, su integridad y desarrollo durante el tiempo de actividad, así como el hecho de asegurar el cuidado y la preservación del medio ambiente en pos de mantener un control sobre los niveles mínimos y máximos permisibles de elementos que son dañinos para el medio ambiente, ya que, de una forma u otra, termina siendo prejudiciales en igual o mayor medida para el personal que labora en la planta.

De acuerdo con los enfoques recientes en materia de salud y seguridad en el trabajo, se pretende mejorar la eficiencia de la gestión de la seguridad en los entornos de fabricación a través de una mejora continua en términos de la seguridad laboral e industrial por medio de indicadores como son los seis ejes que definen la cultura de la seguridad en una organización: compromiso, coherencia, objetividad, tolerancia, comunicación y apoyos (Brocal, 2019).

A grandes rasgos, se requiere que todos los factores involucrados sean implementados y en conjunto, se logre un equilibrio que permita conseguir la eficiencia en términos de seguridad; por mencionar, se requiere de compromiso y responsabilidad por todas las partes a modo de definir normas que todos deban atender; ser coherentes en todos los aspectos posibles a fin de no cometer imprudencias ni aplicar lógicas irracionales; ser objetivos con los propósitos, no trazar ideales o escenarios fuera de lugar; ser tolerantes ante las deficiencias y promover la capacitación sobre el control y manejo de lo que se esté relacionando; mantener la comunicación en todo momento es primordial para lograr un buen desempeño y finalmente apoyar en todos los sentidos necesarios para cumplir con las tareas bajo el menor riesgo posible (Torrecilla, 2020).

Ya que, según estimaciones recientes de la Organización Internacional del Trabajo (OIT), cada año mueren 2,78 millones de trabajadores por accidentes laborales y enfermedades relacionadas con el trabajo (de los cuales 2,4 millones están relacionados con enfermedades) y otros 374 millones de trabajadores sufren accidentes laborales no mortales. Se torna estrictamente necesario el atender en todo momento, el análisis de riesgos y el acatamiento de las normas y políticas estipuladas (Wadsworth, 2019).

Así pues, dentro de los aspectos que se adaptan al desarrollo y seguimiento de la seguridad industrial, no solo se trata de medidas preventivas, niveles de peligro y asignación de normativas para realizar las tareas sin el riesgo de sufrir lesiones; también resulta importante tomar en cuenta el factor de comodidad y entusiasmo con el que el trabajador va a realizar sus tareas. Es decir, se 
debe considerar que la ergonomía juega un papel especial con el desempeño que el trabajador tendrá en sus actividades conforme el entorno que le es ofrecido, las medidas y consideraciones tomadas a razón de mantener cada factor previsto, bajo control (Gualtieri, 2020).

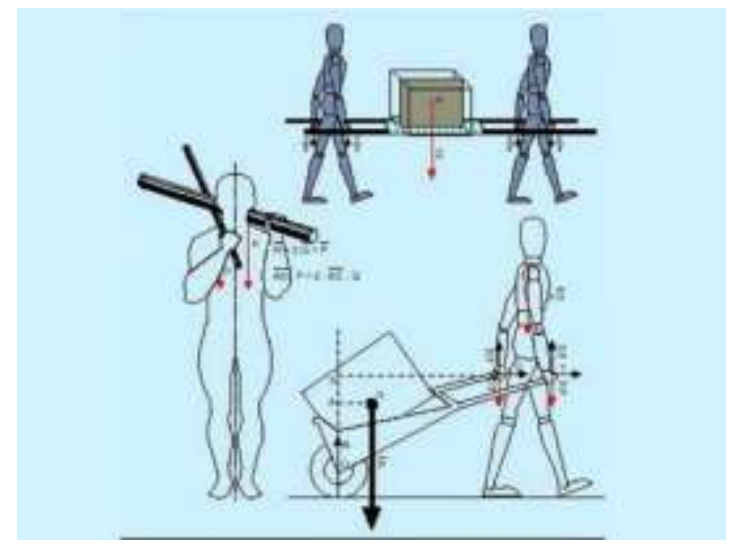

Figura 5. Ergonomía sobre el rendimiento laboral (tomada de Velázquez, 2020)

De modo que, la seguridad y la salud de los trabajadores no es un asunto del que se pueda prescindir, ya que son los trabajadores quienes aumentan la productividad y conducen a un mejor rendimiento, son ellos con sus habilidades y compromiso laboral quienes mejoran la imagen de la empresa, reducen las reclamaciones y los accidentes al acatar las normas establecidas, haciendo acato de las indicaciones que les son emitidas y también, son plenamente conscientes de los tiempos perdidos por lesiones y pérdidas materiales que pueden ser ocasionados tras un mal desempeño en las actividades que les son encomendadas (Mashwama, 2019).

\section{Conclusión}

A grandes rasgos, los eventos que dieron pie al desarrollo y aplicación de medidas que surtieran efecto en disminuir la causalidad de accidentes y enfermedades entre los trabajadores, tomaron fuerza con el paso de cada evento que ha quedado marcado en la memoria de la humanidad. Sobresaliendo como una pequeña lucha que día a día se fue acrecentando hasta convertirse en una imagen obligatoria con la que todo establecimiento debe contar. No obstante, aún con la importancia que ha ganado la seguridad y salud ocupacional, prevalecen idealismos que carecen de sentido común y que, desafortunadamente, comienzan a ser aceptado.
Así pues, considerando que los accidentes laborales se han vuelto parte de un entorno diario y que se definen ya como un suceso normal, se ha dado pie a que la misma sociedad los vea con tranquilidad, asumiendo que todo trabajo conlleva un riesgo y que, por tanto, nadie está libre o menos propenso de sufrir un accidente. De este modo, es preciso declarar que todo termino ambiguo con relación a la seguridad industrial queda descartado y se da paso a un panorama más concreto; así pues, se puede enunciar que lo relacionado a seguridad industrial e higiene ocupacional se encuentra ligado a todos aquellos factores inherentes al entorno que rodea la actividad laboral; haciendo referencia a condiciones ambientales, zonas restringidas, ventilación, iluminación, control y resguardo de maquinaria y herramientas, manejo de sustancias, uso de equipo de protección personal, espacios libres de contaminación, control sanitario y otro tipo de factores que se destacan en brindar al personal, la mayor seguridad en su estancia durante sus actividades laborales.

Con el pasar de los años, se ha demostrado que el ser humano siempre buscará mejorar sus condiciones, adquirir conocimiento y volverse capaz y habilidoso en el desempeño de sus acciones; esto deriva de conjugar la parte práctica con la postura moral y cultural de las personas, ya que las empresas, como una entidad presencial ante el sector industrial, no son las únicas responsables de asegurar el cuidado y la preservación tanto de la vida humana como del medio ambiente; es cierto que deben proveer al personal de todo lo necesario para brindar seguridad a sí mismo y al resto de personal que labora en una industria o empresa de servicios, pero también, es parte fundamental que el empleado como entidad individual, asuma su papel con responsabilidad y compromiso, asegurando que su comportamiento será el adecuado para dar plena seguridad de que sus acciones no le causen algún accidente a el mismo o a otras partes del personal.

Por otro lado, un factor preliminar que ata a la sociedad al caos constante es la sobreestimación. Como seres racionales se debe entender una simple y sencilla cosa: los humanos no son seres inmortales o indestructibles, nadie en el conocido plano terrenal está absuelto de no correr peligro inminente; se es fuerte y débil por igual. Sin embargo, resulta complicado asumir la debilidad como algo objetivo, normalmente se atribuye a un enfoque más emocional que físico; por ello, es preciso decir que ante tal idea de superioridad, se permite encontrar el origen para las acciones tan incoherentes y desmedidas que muchos trabajadores suelen tomar. Por mencionar algunas, creen tener control absoluto de su equilibrio y confían plenamente en sus habilidades de maniobra 
como para laborar en tareas a grandes alturas sin estar sujetos a ningún equipo de protección; consideran que no es necesario utilizar casco de protección en una obra subterránea porque están seguros de que no corren peligro alguno o que, en dado caso de que ocurra un derrumbe, serán lo suficientemente rápidos para ponerse a salvo; no miden la dimensión de los riesgos que se encuentran latentes y que en cualquier momento pueden tomar su vida por su falta de buen juicio.

En palabras comunes, el ser humano carece de un cuerpo resistente como el acero, no está hecho de ningún tipo de material que se vuelva ventajoso para aventajar ante situaciones riesgosas, ninguna acción divina será manifestada para evitar el sufrir una lesión grave o morir por no atender las indicaciones, por no tomar las medidas o por no usar el equipo de protección personal; cada individuo es totalmente capaz y responsable de mantener su propia integridad ya que nadie más lo hará en su lugar. El carácter multidisciplinar de la seguridad y salud ocupacional explica la diversidad de disciplinas que se han involucrado en esta área de investigación. En donde los tópicos más relevantes están relacionados con salud ocupacional, responsabilidad social corporativa, sostenibilidad, seguridad, desempeño, administración y estrés ocupacional. En donde las áreas de investigación más productivas en este tema son: ciencia y tecnología, ingeniería, ecología, investigación de operaciones, negocios y economía. Es de destacar que, entre las áreas de investigación analizadas en esta revisión documental, el sector de la salud es el que menos atención o aportación bibliográfica ha realizado sobre seguridad y salud ocupacional.

Sin mencionar que, de manera sustancial, las aportaciones no han sido del todo acrecentadas puesto que, como bien se ha mencionado anteriormente, los campos de estudio han puesto sus ojos en elementos más estrictos en cuanto a dominio de conocimiento; siendo así, es preciso denotar que de forma clara y hasta cierto punto injusta, el estudio a profundidad sobre temas relacionados con la seguridad y salud ocupacional se ve limitado por una razón sustentada en lo irracional; todo lo relacionado al cuidado y protección es un punto que todo individuo debe comprender y aplicar, por el simple hecho de ser una parte instintiva que no requiere de mayor enfoque $o$ análisis ya que carece de aspectos que deban ser desentrañados, cosa que es totalmente errónea; todo conocimiento debe ser abordado.

Esta investigación documental ha mostrado que los nuevos desarrollos científicos y tecnológicos de la cuarta revolución industrial aportarán numerosas ventajas para diseñar arquitecturas (Sistema de Gestión de Seguridad y Salud en el Trabajo) más robustas que permitan seleccionar variables más relevantes en esta disciplina con el objetivo de recopilar, manejar y analizar información en tiempo real para predecir y prevenir accidentes laborales. Sin embargo, este paradigma también conlleva riesgos y desafíos emergentes relacionados con el desempeño organizacional y humano. Estos riesgos emergentes incluyen tanto riesgos laborales como riesgos industriales. Podría decirse que el factor humano es el vínculo principal entre los riesgos emergentes industriales y los riesgos emergentes ocupacionales en el contexto de la Industria 4.0.

\section{Referencias}

Adem A, Çakit E, Dağdeviren M. Occupational health and safety risk assessment in the domain of Industry 4.0. SN Appl Sci [Internet]. 2020;2(5). Disponible en: http://dx.doi.org/10.1007/s42452-020-2817$\mathrm{x}$.

Ajayi A, Oyedele L, Davila Delgado JM, Akanbi L, Bilal M, Akinade O, et al. Big data platform for health and safety accident prediction. World J Sci Technol Sustain Dev. 2019; 16(1): 2-21.

Antezana, PG. (2012). Historia del derecho laboral. Fides et Ratio Revista de Difusión cultural y científica de la Universidad La Salle en Bolivia, 5(5), 67-78. Recuperado el 23 de marzo de 2021, de http://www.scielo.org.bo/scielo.php?script=sci_arttext\&pid=S2071081X2012000100007\&lng=es\&tlng=es.

Arias GWL. Revisión histórica de la salud ocupacional y la seguridad industrial. Revista Cubana de Salud y Trabajo. 2012;13(3): 45-52.

Badri A, Boudreau-Trudel B, Souissi AS. Occupational health and safety in the industry 4.0 era: A cause for major concern? Saf Sci. 2018; 109: 403-11.

Bernal Mora, Héctor, La explicación de la humanidad del hombre. el origen del carácter de producción, del sentimiento de separatidad, de la conciencia desarrollada. la selección innatural. Nómadas. Critical Journal of Social and Juridical Sciences [Internet]. 2012;34(2): Recuperado de: https://www.redalyc.org/articulo.oa?id=18126057014.

Bortolini M, Botti L, Galizia FG, Mora C. Safety, ergonomics and human factors in reconfigurable manufacturing systems. En: Springer Series in Advanced Manufacturing. Cham: Springer International Publishing; 2020: 123-38.

Brocal F, González C, Komljenovic D, Katina PF, Sebastián MA. Emerging risk management in Industry 4.0: An approach to improve organizational and human performance in the complex systems. Complexity. 2019; 2019: 1-13.

Espantelon, A. (2013, 11 septiembre). Gestión integrada de la calidad, medio ambiente y seguridad y salud en el trabajo. Parte III: PDCA Home. Recuperado el 25 de Marzo de 2021, de https://www.pdcahome.com/5411/gestion-integrada-de-la-calidadmedio-ambiente-y-seguridad-y-salud-en-el-trabajo-parte-iii/.

Fan D, Zhu CJ, Timming AR, Su Y, Huang X, Lu Y. Using the past to map out the future of occupational health and safety research: where do we go from here? int j hum resour manag. 2020; 31(1): 90-127

Fresquet, F. J. L. (2011, 12 febrero). Bernardino Ramazzini (1633-1714). Medicina, Historia y Sociedad. https://historiadelamedicina.wordpress.com/2011/02/12/bernardinoramazzini-1633-1714/.

Gnoni MG, Bragatto PA, Milazzo MF, Setola R. Integrating IoT technologies for an "intelligent" safety management in the process industry. Procedia Manuf. 2020;42:511-5.

Gualtieri L, Palomba I, Wehrle EJ, Vidoni R. The opportunities and challenges of SME manufacturing automation: Safety and ergonomics in human-robot collaboration. En: Industry 40 for SMEs. Cham: Springer International Publishing; 2020: 105-44. 
Hofmann DA, Burke MJ, Zohar D. 100 years of occupational safety research: From basic protections and work analysis to a multilevel view of workplace safety and risk. J Appl Psychol. 2017; 102(3): 375-88.

Hudson D, Ramsay JD. A roadmap to professionalism: Advancing occupational safety and health practice as a profession in the United States. Saf Sci. 2019; 118: 168-80.

Mashwama N, Aigbavboa C, Thwala W. Occupational health and safety challenges among small and medium sized enterprise contractors in South Africa. En: Advances in Intelligent Systems and Computing. Cham: Springer International Publishing; 2019. p. 68-76.

Ncube F, Kanda A. Current status and the future of occupational safety and health legislation in low- and middle-income countries. Saf Health Work. 2018; 9(4): 365-71.

Perl J. Environment, safety, and occupational health (ESOH) regulations, En: Sustainability Engineering. Cham: Springer International Publishing; 2016: 75-86.

Polak-Sopinska A, Wisniewski Z, Walaszczyk A, Maczewska A, Sopinski P. Impact of industry 4.0 on occupational health and safety. En: Advances in Manufacturing, Production Management and Process Control. Cham: Springer International Publishing; 2020. p. 40-52.

Ramos D, Afonso P, Rodrigues MA. Integrated management systems as a key facilitator of occupational health and safety risk management: A case study in a medium sized waste management firm. J Clean Prod. 2020;262(121346):121346.

Reis C, Oliveira C, Braga P, Silva JF, Silva LT. Occupational health and safety-sustainable development and the changes in organizations. En Occupational and Environmental Safety and Health II. Cham: Springer International Publishing; 2020: 677-87.

Romero D, Mattsson S, Fast-Berglund Å, Wuest T, Gorecky D, Stahre J Digitalizing occupational health, safety and productivity for the operator 4.0. En: Advances in Production Management Systems Smart Manufacturing for Industry 40. Cham: Springer International Publishing; 2018: 473-81.

Ruiz AM, Choroco V. Realidad en Perú de la gestión de seguridad y salud en el trabajo. 2019. Recuperado el 25 de marzo de 2021, de https://www.marsh.com/pe/es/insights/risk-in-context/gestionseguridad-salud-trabajo.html

Salvador LR, Van Thinh D. Occupational safety and health: An overview. En: 2016 IEEE 11th International Symposium on Applied Computational Intelligence and Informatics (SACI). IEEE; 2016.

Singh V, Singh A, Kaur P. Key parameters of occupational safety for sustainable manufacturing units: A review. En: Advances in Intelligent Systems and Computing. Cham: Springer International Publishing; 2016: 53-61.

Tarrahi M, Shadravan A. Advanced big data analytics improves HSE management. En: Day 1 Wed, April 20, 2016. SPE; 2016.

Thibaud M, Chi H, Zhou W, Piramuthu S. Internet of Things (IoT) in highrisk Environment, Health and Safety (EHS) industries: A comprehensive review. Decis Support Syst. 2018; 108: 79-95.

Torrecilla García JA, Del Carmen Pardo-Ferreira M, Rubio-Romero JC. Prospective analysis of blockchain applications within the occupational health and safety management and wearable-related ergonomics in manufacturing industry. En: Occupational and Environmental Safety and Health II. Cham: Springer International Publishing; 2020: 733-41.

Wadsworth E, Safety of health at the heart of the future of work. Building on 100 years of experience. 2019. p. 82. 